\title{
Design and Simulation of Novel MEMS based Capacitive Microphone
}

\author{
Lourembam Bandana ${ }^{1}$, V. S. Selvakumar ${ }^{2}$ \\ ${ }^{1}$ PG Scholar, Department of ECE, Rajalakshmi Engineering College, Chennai, Tamil Nadu \\ ${ }^{2}$ Associate Professor, Department of ECE, Rajalakshmi Engineering College, Chennai, Tamil Nadu
}

\begin{abstract}
Nowadays, MEMS (Micro-electromechanical Systems) technology is widely used to design various types of microphone. The capacitive microphone is highly preferable because of its high achievable, sensitivity, miniature size, batch fabrication, integration feasibility and long stability performance. In this paper, a novel MEMS capacitive microphone operating at a low actuating voltage is presented. It consists of Aluminum diaphragm arranged over the rigid Silicon back plate in which the diaphragm includes a number of holes to reduce the acoustical damping as well as stiffness. The design and simulation has been done using the three modules of Intellisuite- Intellimask, 3D builder and TEM (Thermo-electromechanical) analysis. Comparing the diaphragm of two different thicknesses, the thinner one gives higher deflection under the same applied pressure. The variation in the capacitance with the increase in pressure is analysed.The simulated result shows that the pull-in Voltage is $0.3 \mathrm{~V}$ and the zero bias capacitance is 5.09 Pf. A process flow for the fabrication is designed and simulated using Intellifab.
\end{abstract}

Keywords: MEMS, microphone, TEM, Pull-In voltage, Intellifab

\section{Introduction}

MEMS microphone is a transducer that converts acoustic energy into electrical energy. Some of its application includes voice communication device, hearing aids, surveillance and military aims, ultrasonic and acoustic distinction under water and noise and vibration control [1]. In MEMS Capacitive microphone design, it is important to focus on small size and high mechanical sensitivity as the two most desirable factors. Mechanical sensitivity is determined by the material properties (such as Young's modulus and the Poisson's ratio), thickness and intrinsic stress in the diaphragm.

Many researchers have investigated the fabrication of MEMS microphone by selecting different structures and materials to optimize the sensitivity. Most surfaced and bulk micro machined capacitive microphone uses fully clamped diaphragm with perforated back plate [6].The fabrication process involved are very long, cumbersome, expensive and not compatiblewith high volume process. Moreover they are not small in size. Many traditional MEMS capacitive microphone are fabricated in high temperature [7] where the structure is easily damaged. A condenser microphone is proposed with a $\mathrm{p}^{+}$silicon membrane without acoustic holes [8]. Because of the absence of holes, the air gap is increased to $7.5 \mu \mathrm{m}$ in order to alleviate the acoustical damping.

In this work, we proposed a novel MEMS capacitive microphone, which uses perforated Aluminum diaphragm to obtain small size, low cost and improved microphone sensitivity. The process requires the design of only three masks layers thereby making it simple and easy to fabricate.

\section{Design Aspects}

The structure of the microphone consists of three layers as shown in figure 1. The topmost layer consists of flexible Aluminum diaphragm, anchor made out of Silicon dioxide as the insulating layer and the fixed bottom Silicon electrode. The diaphragm includes a number of holes to allow the air in the gap between the diaphragm and the lower electrode to escape and thus reduce acoustical damping in the microphone.

When the diaphragm is exposed to the acoustic wave, it causes flexural vibration and changes the average distance from the back plate. This in turn will produce a change in capacitance giving rise to a time varying voltage on the electrodes. The static capacitance s given by

$$
C=\varepsilon_{\circ} \frac{A}{d}
$$

Where $\varepsilon_{\circ}$ is the dielectric constant of the air and $A$ is the surface area of the membrane.

In order to actuate the microphone, it should be provided with a DC bias voltage across the capacitor structure. The electrostatic force is induced by the bias voltage across the two electrodes which is given by

$$
F_{S}=\frac{v^{2} \varepsilon_{\mathrm{o}} A}{2 g^{2}}
$$

Where $\mathrm{V}=$ applied voltage

$\varepsilon_{\circ}=$ permittivity of free space

$\mathrm{g}=$ gap between the electrodes

$\mathrm{A}=$ area of the surface electrode

In order to optimize the sensitivity of the microphone, the design parameters used for the proposed structure are shown in table 1

Table 1:Design parameters

\begin{tabular}{|c|c|}
\hline Parameters & Value \\
\hline Diaphragm material & Aluminum \\
\hline Diaphragm area & $1 \mathrm{mmx} 1 \mathrm{~mm}$ \\
\hline Diaphragm thickness & $3 \mu \mathrm{m}$ \\
\hline Air gap & $2 \mu \mathrm{m}$ \\
\hline Number of holes & 25 \\
\hline Back plate thickness & $10 \mu \mathrm{m}$ \\
\hline Acoustic hole size & $50 \mu \mathrm{m} \times 50 \mu \mathrm{m}$ \\
\hline
\end{tabular}




\section{International Journal of Science and Research (IJSR) \\ ISSN (Online): 2319-7064}

Index Copernicus Value (2013): 6.14 | Impact Factor (2014): 5.611

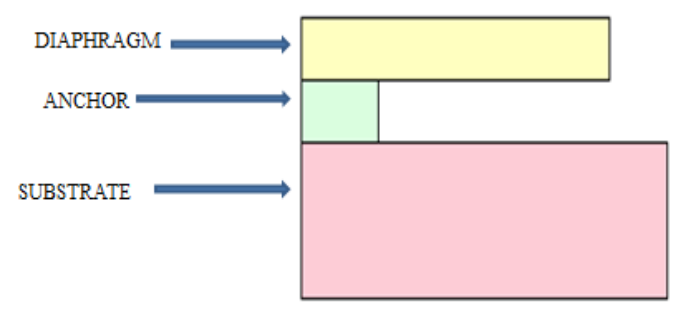

(a) Side View

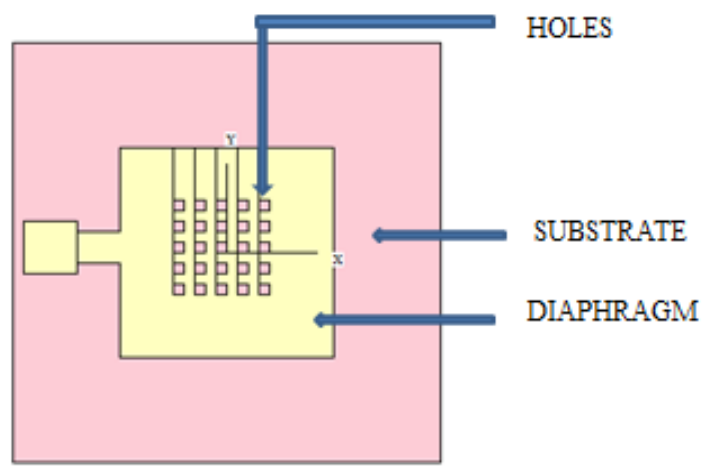

(b) Top View

Figure 1: Structure of the microphone

\section{Simulation}

The three masks layers in 2D structure is created using the Intellisuite module (Intellimask), which is imported to the $3 \mathrm{D}$ builder in figure 2 where the thickness of the layers and different entities are defined. Next, the 3D structure is imported to TEM (Thermo-electromechanical) module where the material types, boundary and loads are defined. Finally, TEM analysis is performed to obtain the final simulated results.

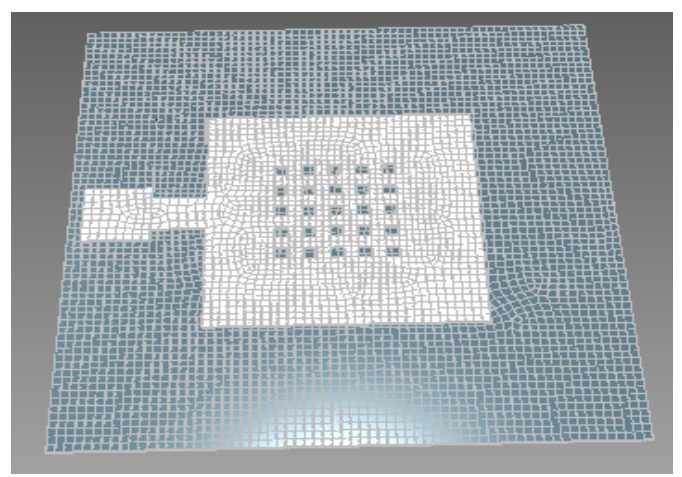

Figure 2: 3D View of the structure

\subsection{Pressure versus Displacement}

The required voltage of around $0.29 \mathrm{~V}$ is applied to the lower and upper electrodes of the structure while a pressure of 0.7 $\mathrm{Pa}$ is applied to the top surface of the diaphragm. The resultant deformation of the diaphragm is shown in figure 3 , which is taken along the Z-axis.

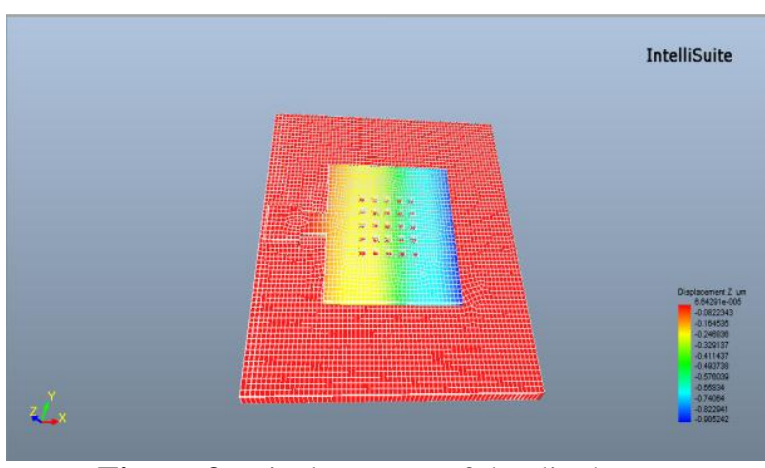

Figure 3: Displacement of the diaphragm

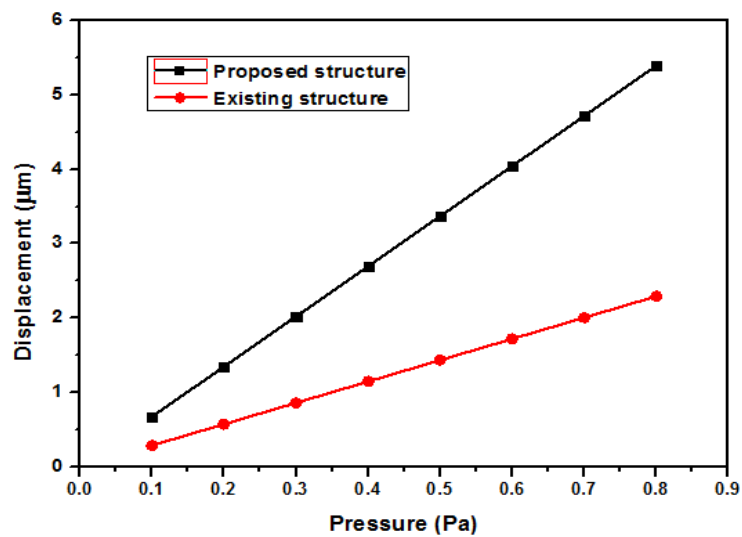

Figure 4: Graph of pressure versus Displacement for two different diaphragms

The same analysis is done for the diaphragm of different thicknesses of $3 \mu \mathrm{m}$ and $4 \mu \mathrm{m}$ respectivelyfor the pressure ranging from $0.1 \mathrm{~Pa}$ to $0.8 \mathrm{~Pa}$. It is found that the thinner diaphragm shows greater deflection in comparison with the thicker one. The higher deflection in turn gives better sensitivity of the microphone. The displacement increases linearly with the pressure in figure 4 .

\subsection{Pressure versus Capacitance}

When the diaphragm is applied with different load pressure ranging from $0 \mathrm{~Pa}$ to $0.07 \mathrm{~Pa}$, there is change in the capacitance value due to deformation. The graph is plotted between the pressure and the corresponding capacitance as shown in figure 5 . The result shows that the capacitance increases non linearly with the increase in pressure.

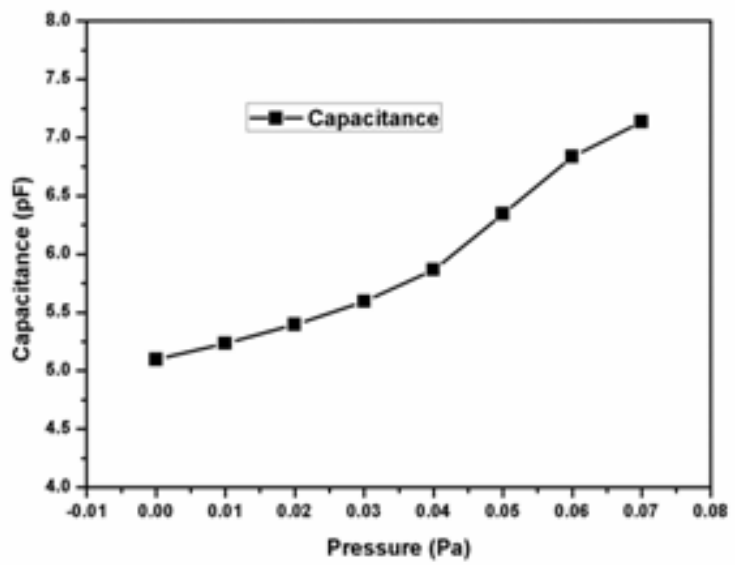

Figure 5: Graph between Pressure and Capacitance 


\section{International Journal of Science and Research (IJSR) \\ ISSN (Online): 2319-7064 \\ Index Copernicus Value (2013): 6.14 | Impact Factor (2014): 5.611}

\subsection{Voltage versus Capacitance}

When the applied voltage is increased, the electrostatic force of attraction between the upper and the lower electrode increases and pulls the beams towards the bottom elctrode. This leads to an increase in the resulted capacitance due to the variable gap distance. When the height of the diaphragm reaches $\frac{2}{3}$ of the initial gap, the electrostatic force becomes greater than the natural restoring force and the beam collapses to touch the bottom electrode. The voltage at this point is called "pull-In voltage" or "collapse voltage",

$$
V=\sqrt{\frac{8 K g_{\mathrm{o}}{ }^{3}}{27 \varepsilon_{\mathrm{o}} A}}
$$

The simulated graph of voltage Vs Displacement along the $\mathrm{Z}$-axis is auto generated in the Intellisuite software as shown in figure 6.

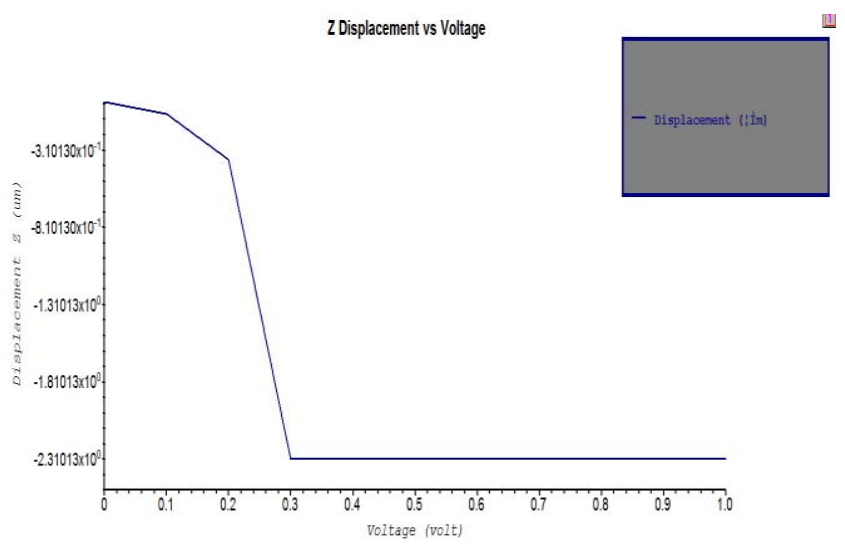

Figure 6: Graph of Voltage versus Capacitance

It is seen that the displacement increases with the increase in voltage applied upto a certain point and remains constant.From this, it can be inferred that the pull-In voltage occurs at $0.3 \mathrm{~V}$ after which the diaphragm membrane snapped to the bottom surface.

\section{Fabrication}

A process flow is designed for the fabrication of the proposed microphone on a Silicon substrate. The proposed fabrication process is designed using three masks. The masks for the design are generated in Intellimask. Mask-0 is used for the Silicon substrate, mask-1 is used for the anchor part Silicon dioxide and mask-2 is used to create the Aluminum diaphragm membranes with holes. The process flow is designed and simulated using Intellifab/ Fabviewer by importing the mask layout from the Intellimask file. The important stages of the process flow are shown in figure 7.

The fabrication process starts with the definition of Silicon substrate on top of which $2 \mu \mathrm{m}$ Silicon dioxide is deposited as shown in figure 7 (a). A positive photoresist is applied followed by UV exposure of the surface to pattern mask-1 in figure 7(b). Etching of the Silicon dioxide followed by Reactive Ion etching leads to the formation of anchor part in figure $7(\mathrm{c})$. An Aluminum layer of thickness $3 \mu \mathrm{m}$ is deposited on top of the PSG layer in figure 7(d), which will form the flexible diaphragm and patterned using mask-2 in figure 7 (e). To create the gap between the diaphragm and the bottom electrode, a sacrificial layer of PSG is deposited which is etched out in the later part. Finally, the desired structure is released by etching off the sacrificial layer. The top view of the final structure is as shown in figure 7(f).

The resulting steps of fabrication are as follows:

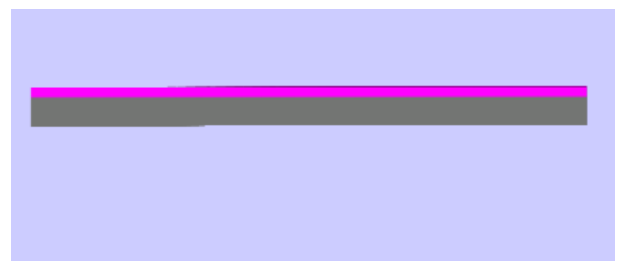

a) Deposition of $\mathrm{SiO}_{2}$ on the $\mathrm{Si}$ substrate

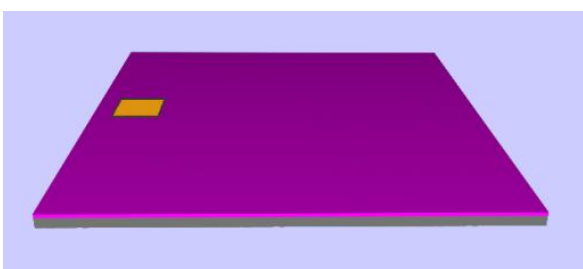

b) Deposition of the positive photoresist and UV exposure for mask 1

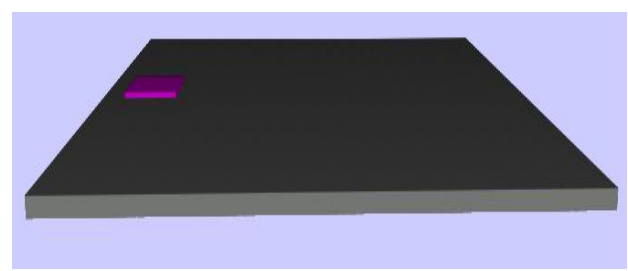

c) Etching to form the anchor part $\mathrm{SiO} 2$

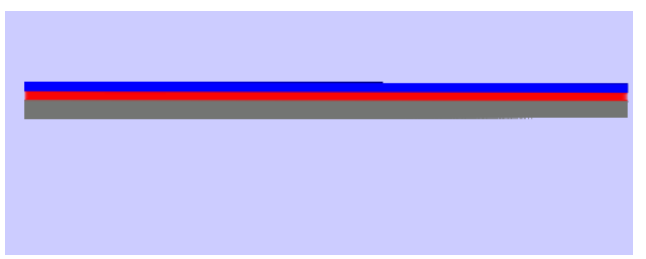

d) Deposition of PSG and Al for the diaphragm

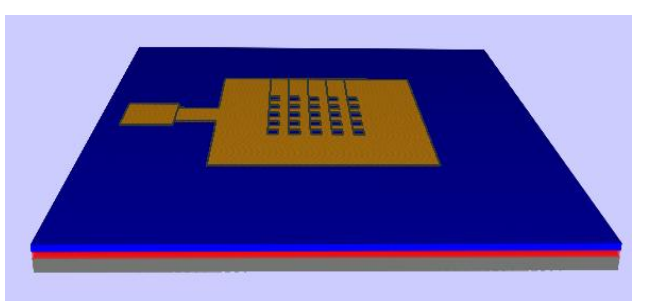

e) Deposition of the positive photoresist and UV exposure for mask 2

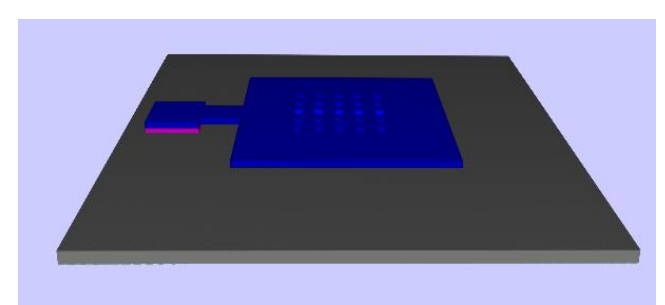

f) Final etching to release the perforated diaphragm

Figure 7: Process flow for the design simulated in Intellifab 


\section{International Journal of Science and Research (IJSR) \\ ISSN (Online): 2319-7064}

Index Copernicus Value (2013): 6.14 | Impact Factor (2014): 5.611

\section{Conclusion}

In this paper, a novel MEMS Capacitive microphone operating at a lower voltage is successfully designed and simulated. The design is simple and easy to implement since it uses only three mask layers. The simulated result shows that thin diaphragm has greater displacement which in turn gives better sensitivity. The pull-In voltage obtained using analytical calculation is $0.39 \mathrm{~V}$ and the simulated results shows $0.3 \mathrm{~V}$. A process flow for the fabrication has been designed and simulated using Intellifab.

\section{References}

[1] Ma.T.Man.T.Y.Chana,Y.C.Zohar,Y.Wong.M,"Design and Fabrication of an IntegratedProgrammable floating gate microphone," Proceedings of the Fifteenth International Conference on Microelectromechanical systems,pp.288-291

[2] Jing C., Liu.L.,Li,Z,Tan,Z,Xu,X.Ma,J,(“On the singlechip condenser miniature microphone using DRIE and backside etching techniques, "SensorsActuators, A 103.42-47,2003

[3] Pappalardo.M.A Caronti,“A new approach to ultrasound generation of the capacitive micro machinedtransducers, "University Rome,Italy,2002

[4] Kabir A.E.,Bashir,R.Bernstein.J.,De Sntis,J.Mathews R.O Boyle J.O Bracken.C,"Very high sensitivity acoustic transducers with thin membrane and back plate, "Sensors and Actuators-A,78:138-142,1999

[5] Kronast, W,Muller,B.Siedel,W.Stoffel.A, "Single chip condenser microphone using porous Silicon as sacrificial layer for the air gap,"Sensors,Actuators,A87:188-193,2001.

[6] Ning,JLiu.H.GeY, “ A Silicon Capacitive microphone based on oxidized porous Silicon sacrificial tehnology," Proceedings $7^{\text {th }}$ international Conference on solid state and Integrated Circuits Technology,IEEE, 3:1872-1875

[7] Chowdhury,S,Jullien,G.A,Ahmad,M.A.,Miller,W.C,“ MEMS acousto-magnetic components for use in a hearing instrument, "presented at SPIE's Symposium on Design test Integration and Packaging of MEMS/MOEMS,Paris,2000.

[8] T.Bourouina, S.Spirkovitch,F.Bailieu and C.Vauge,“ A new Siliconcondensermicrophonewith $\mathrm{p}^{+}$ Siliconmembrane ," Sensors and Actuators A,31,149152,1992

[9] Rombach, P., Mulluborer, M.Klein, U.,RasmussenK "The first low voltaje low noise differential Silicon microphone technology development and measurementresults," Sensors,Actuators,A95:196201,2002.

[10] N.Bilaniuk,“Opticalmicrophonetransductiontechniques, "Applied Acoustics.Vol.50.no.1,pp.35-63,1997.

[11] S.Chowdhury,M.Ahmadand W.C. Miller, " Non linear effects in MEMS Capacitivemicrophonedesign," Proceedings International Conferenceon MEMS Nano and Smart systems,pp.297-302.

[12] TaiRanHsu,"MEMS and Microsystems: Design and Manufacture,"Mc Graw-Hill publications

[13] P.R.Scheeper,A.G.H Van Donk,W.Olthius, “A review of Silicon microphones," Sensor and Actuators,A,44,PP.1-11,1994

\section{Author Profile}

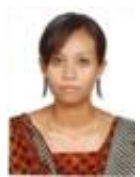

Lourembam Bandana graduated from Vels University; Chennai in the year 2013.She received her master's degree from Rajalakshmi Engineering College, Chennai. She has attended workshops on MEMS and worked in National MEMS Design Centre, REC, Chennai.

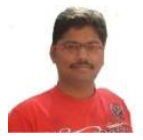

V.S Selvakumarreceived his Master's degree from Satyabhama Institute of Science and technology, Chennai in 2005. He is currently working as associate professor in the Department of Electronics and Communication Engineering, Rajalakshmi Engineering College. $\mathrm{He}$ is also pursuing his Ph.D. in MEMS at National MEMS Design Centre, REC, Chennai 